\title{
HYBRID CELL SELECTION-BASED HEURISTIC FOR CAPACITATED MULTI-FACILITY WEBER PROBLEM WITH CONTINUOUS FIXED COSTS
}

\author{
Nur Shifa Farah Ain Jamil ${ }^{1}$, Syariza Abdul-Rahman ${ }^{1, *}$, Martino Luis $^{2}$ \\ And Aida Mauziah Benjamin ${ }^{1}$
}

\begin{abstract}
Location-allocation problem (LAP) has attracted much attention in facility location field. The LAP in continuous plane is well-known as Weber problem. This paper assessed this problem by considering capacity constraints and fixed costs as each facility has different setup cost and capacity limit to serve customers. Previous studies considered profitable areas by dividing continuous space into a discrete number of equal cells to identify optimal locations from a smaller set of promising locations. Unfortunately, it may lead to avoid choosing good locations because unprofitable areas are still considered while locating the facilities. Hence, this allows a significant increment in the transportation costs. Thus, this paper intelligently selected profitable area through a hybridization of enhanced Cell Selection-based Heuristic (CSBH) and Furthest Distance Rule (FDR) to minimize total transportation and fixed costs. The CSBH divides customer distribution into smaller set of promising locations and intelligently selected profitable area to increase possibility of finding better locations, while FDR aims to forbid the new locations of the facilities to be close to the previously selected locations. Numerical experiments tested on well-known benchmark datasets showed that the results of hybrid heuristic outperformed single CSBH and FDR, while producing competitive results when compared with previously published results, apart from significantly improving total transportation cost. The new hybrid heuristic is simple yet effective in solving LAP.
\end{abstract}

Mathematics Subject Classification. 90C59, 90C27, 68T20, 90B06.

Received October 7, 2020. Accepted May 3, 2021.

\section{INTRODUCTION}

Location-allocation problem (LAP) is a class of facility location problem that has been studied for half a century due to its wide practical application backgrounds. The study on location problem was formally introduced by Weber in 1909, in which the location of a single warehouse was considered to minimize the total distance between warehouse and customers [30]. The aim of the location problem is to locate facilities to efficiently supply to customers. In addition, LAP is a twofold problem as it locates facilities and allocates demand points to the facilities simultaneously. The optimum location-allocation of the facilities and the customer demands are assumed to improve the efficiency of service provided by the facilities [12].

Keywords. Location-allocation problem, capacitated Weber problem, fixed cost, cell-based approach, Furthest Distance Rule.

1 School of Quantitative Sciences, College of Arts \& Sciences, Universiti Utara Malaysia, UUM Sintok, Kedah 06010, Malaysia.

2 College of Engineering, Mathematics, and Physical Sciences, University of Exeter, North Park Road, Exeter EX4 4QF, UK.

* Corresponding author: syariza@uum.edu.my 
Generally, the LAP is divided into two main areas; continuous and discrete models. In solving this LAP, the demands can be satisfied by single or multiple facilities. Thus, continuous LAP served by multiple facilities is also known as multi-facility Weber problem where the facilities can be located anywhere in a continuous plane.

In terms of serving ability of the facilities, the problem may be classified as capacitated or uncapacitated. For the capacitated problem, it refers to facilities with limited capacities. In this case, some demands from customers may be served automatically by the farther facilities because the nearest facilities cannot satisfy their demands due to capacity restrictions. On the contrary, uncapacitated problem refers to facilities with unlimited capacities and the customers are served by the nearest facilities.

Another classification of facility location problem is to consider facility fixed cost. Luis et al. [26] defined fixed cost as a set up or opening cost of a facility that may be dependent according to the geographical area. This may happen as government applies different taxes on urban, suburban, and remote regions or regional restriction lands as some areas are under government protection, such as national parks and heritage sites. In their study, the number of facilities located were either specified or unknown (as a decision variable).

The LAP is proven to be NP-hard where a large-size problem is difficult to be solved by using exact methods [38]. Thus, most of the algorithms to tackle LAP are heuristic-based methods which can only provide good solutions instead of the optimal solution with less computational effort than the exact methods. Brimberg et al. [9] showed that heuristic methods were proven to be the best methods to solve larger NP-hard problems in facility location problems.

Cell-based approach (CBA) and Furthest Distance Rule (FDR) have been used previously to solve the LAP $[14,15,24]$. The CBA is proposed by dividing continuous space into a discrete number of equal cells to identify most or maybe all optimal locations from a smaller set of promising locations. Unfortunately, this approach may lead to avoid choosing good locations from profitable area because unprofitable areas are still considered while locating the facilities. In $[14,15,24]$, all smaller sets of promising locations displayed the possibility to obtain optimal facilities' location. Since the unprofitable areas were also considered, hence it contributes to the increment of the total transportation costs. Thus, this study proposes an enhanced CBA that divides the distribution of customers into smaller set of promising locations and intelligently selects profitable area to increase the possibility of finding better locations. On the other hand, FDR forbids new locations of the facilities to be close to the previously selected locations. This paper, thus, developed a hybridization of enhanced CBA called Cell Selection-based Heuristic (CSBH) and FDR to solve capacitated multi-facility Weber problem in the presence of continuous fixed cost. Next, the performance of the hybrid heuristic was assessed by using a wellknown dataset from the literature and was compared with single CSBH and FDR as well as previous published results.

The rest of the paper is organized as follows. Section 2 reviews the relevant literature on the problem. Section 3 discusses the proposed hybrid heuristic algorithm in more detail. Then, the discussion of the results and comparison are presented in Section 4. Finally, Section 5 concludes this paper and highlights some future research avenues in this area.

\section{LiterATURE REVIEW}

The LAP is a class of facility location that has been studied for half a century due to its wide application in practice. This section reviews the previous relevant literature on capacitated multi-facility Weber problem, also known as continuous capacitated LAP (CCLAP) and followed by the review on the problem in the presence of fixed costs.

\subsection{The continuous capacitated location-allocation problem}

Cooper [11] was the first to study CCLAP and designed both exact and heuristic methods to solve the problem. For the exact method, all basic feasible solutions were produced using the transportation problem to allocate customers to the nearest facilities in order to minimize transportation costs. In the heuristic method, 
alternating transportation-location (ATL) heuristic was proposed to solve LAP and the transportation problem was simultaneously enhanced until no improvement in the total cost was obtained.

Sherali and Tuncbilek [35] re-examined the problem using a distance proportional to the square of the Euclidean distance, apart from developing a branch and bound algorithm to compute strong upper bounds via Lagrangean relaxation scheme. Sherali et al. [36] formulated a mixed integer nonlinear programming for the rectilinear distance CCLAP and put forward a reformulation-linearization technique (RLT) to deal with the problem. Sherali et al. [37] studied the CCLAP with Euclidean and lp distances using the branch and bound technique to establish global optimization procedures.

Zainuddin and Salhi [42] designed a perturbation-based heuristic to solve the CCLAP by considering borderline customers whose locations lie approximately half-way between their nearest and their second nearest facilities. Aras et al. [4] tackled the CCLAP with Euclidean, squared Euclidean, and lp distances using three heuristics which are Lagrangean heuristic, discrete $p$-capacitated facility location heuristic identical to the $p$ median method of [16], and cellular heuristic of [15]. Aras et al. [5] applied simulated annealing, threshold accepting, and genetic algorithms to deal with the CCLAP with rectilinear, Euclidean, squared Euclidean, and lp distances. In a subsequent work, Aras et al. [6] modified their earlier approaches to tackle the rectilinear CCLAP.

Luis et al. [24] introduced region-rejection based heuristics to restrict locating new facilities, which are too close to the previously selected locations. A discretization technique, known as a cell-based approach, was also put forward to convert a plane into a discrete space. Mohammadi et al. [30] dealt with the CCLAP by proposing two genetic algorithms (GAs), one for the location problem and the other for the allocation of customers to these facilities. Luis et al. [25] designed a guided reactive greedy randomized adaptive search procedure by combining an adaptive learning with the concept of region-rejection based heuristics. Akyüz et al. [1] developed two types of branch and bound algorithms where the first is on the allocation space whereas the second is on the partition of the location space to tackle CCLAP. Luis et al. [27] put forward a two-phase technique to deal with the CCLAP where in the first phase, a technique that discretizes continuous space into discrete cells is used to generate initial facility configurations and in phase two, a variable neighborhood search is adopted to improve the final locations.

Several extensions have been integrated to the CCLAP, such as an introduction of stochastic demand. Zhou and Liu [43] studied the CCLAP with stochastic demand and solved the problem by using a hybrid of intelligent algorithm based on the network simplex algorithm, stochastic simulation and genetic algorithm. Hosseininezhad et al. [17] developed a risk management model with customer and financial risks for the CCLAP. Mousavi and Niaki [31] studied the CCLAP with uncertain demand and customer locations by designing a hybrid intelligent algorithm to solve it. Tirkolaee et al. [41] proposed a novel mixed-integer linear programming model for multi-trip location-routing problem with time windows to cater for medical waste of COVID-19 pandemic. The model was tested for a real case study in Sari city of Iran to minimize the total traveling time, total violation from time windows and total risk of infection among population around disposal sites. Sensitivity analyses were also presented to propose useful insight.

Elalouf et al. [13] investigated capacitated problem on the optimization of blood sample collection with timing and quality constraints. The challenge of this study is the clinics where the collected blood samples are geographically dispersed, whereas the availability of testing facilities is limited. Ozgen and Gulsun [34] solved the CCLAP as multi-objective problem by considering possibilistic linear programming and fuzzy AHP that contains quantitative and qualitative factors. Two objective functions related with cost minimization and benefit maximization were optimized based on four-stage supply chain network in the presence of vagueness. Tirkolaee et al. [39] presented a mixed integer linear programming model for the capacitated multi-echelon location inventory problem with the aim to minimize the establishment, transportation, and inventory costs under uncertainty demands. The problem was solved by using exact method and a self-learning particle swarm optimization algorithm, which revealed that the proposed algorithm outperformed the exact method by providing high quality solutions in a reasonable amount of computational time. 
Alizadeh et al. [2] employed a Bernoulli distribution function to solve capacitated multi-facility locationallocation model with stochastic demands. The discrete capacitated sub-sources introduced in the model allowed facilities to cooperate with other sub-sources. Alizadeh et al. [3] then improved their previous work in [2] by formulating the problem as mixed-integer nonlinear programming based on a problem at an automobile manufacturer. The proposed problem was transformed from nonlinear to linear constraint for model simplification. The small size problem is able to obtain optimal solution while medium and large problems were solved by using genetic algorithm and a discrete version of the colonial competitive algorithm. The study found that the produced solutions are efficient. The following subsection provides relevant literature in the CCLAP in the presence of fixed costs.

\subsection{The capacitated location-allocation problem with fixed costs}

Brimberg et al. [9] were among the first to study the CCLAP with constant fixed costs and to design a three-phase heuristic. The first phase estimates the number of facilities by solving the discretized problem, followed by basic location improvement by using Cooper's location allocation heuristic, and the final step is solution improvement by using local search with different number of facilities. The proposed approach can efficiently solve the problem. Brimberg and Salhi [7] investigated a single facility in the continuous space with zone-dependent fixed costs. Their results highlighted that the optimal solution fell either inside a zone with the cheapest cost, or on a zone edge. An exact algorithm, based on the above observation, was also discussed.

Luis et al. [26] studied the CCLAP with three different types of facility fixed costs, which are (1) a constant fixed cost function, (2) a zone-based fixed cost function and (3) a continuous fixed cost function. For a constant fixed cost function, the cost components in the objective function are assumed to be equal between each facility and customer pair [9]. In a zone-based fixed cost function model, the plane is divided into mutually exclusive zones and the fixed cost is assumed to be constant within each zone. The disadvantage of this model is a small change in locations that may cause a large change in the fixed costs. Thus, a continuous fixed cost function model is proposed to improve the problem. Continuous fixed cost is defined as the fixed costs of the facilities based on the nearest customers' locations where the fixed cost of the nearest customers' locations is the fixed costs of the facilities.

Hosseininezhad et al. [18] dealt with the CCLAP with fixed costs by considering situations in practice that some zones may have high installation cost to set up a facility. In their study, the fixed cost was defined as a zonebased fixed cost that involved production and installation cost. First, nonlinear and mixed integer formulations were presented and then, three stages of Cross-Entropy metaheuristics with novel density function were proposed. Tirkolaee et al. [40] considered fixed locational costs of collection and processing/disposal facilities together with other costs such as operational costs of facilities, transportation costs, penalty costs of non-collected waste, and costs arising from pollution emissions in a robust green location-allocation-inventory problem for municipal solid waste (MSW) management system. The problem was modelled as mixed-integer linear programming and solved based on real-life data using CPLEX solver of GAMS software under deterministic and uncertain conditions.

Mousazadeh et al. [32] studied a health service network design. The model aims to minimize the total fixed cost and total weighted distance between patient zones and health facilities simultaneously. Thus, the main strategic decisions that must be made are the locations of health service providers, type of services and capacity of provided services, allocation of geographical regions to health service providers, and design of referral system in a hierarchical configuration. Irawan et al. [19] developed a new model for the single source CCLAP with the presence of facility fixed cost which incorporates multi-level type capacity (or design). The fixed cost is based on capacity and zone-dependent with convex polygons using triangular shape. In the following research, [20] designed a matheuristic approach to tackle discrete and continuous LAP with the presence of several possible capacities and the opening (fixed) cost of a facility that is dependent on the capacity used and the area where the facility is located.

Lara et al. [23] solved the capacitated multi-facility continuous LAP with fixed costs using a nonlinear Generalized Disjunctive Programming model. Due to the noncovex problem, a bi-level decomposition algorithm was put forward to solve the linear version of the problem. Irawan et al. [21] studied the capacitated multi-facility 
TABLE 1. Comparison of solution methods used for continuous capacitated LAP.

\begin{tabular}{llll}
\hline \hline \multirow{2}{*}{$\begin{array}{l}\text { Continuous } \\
\text { capacitated LAP }\end{array}$} & \multicolumn{3}{c}{ Solution method } \\
\cline { 2 - 4 } & Exact & Heuristics & Metaheuristics \\
\hline No fixed cost & {$[1,3,11,35-37,39,41]$} & {$[4,11,24,25,42]$} & {$[3,5,6,27,30,31,43]$} \\
Fixed cost & {$[7,40]$} & {$[9]$} & {$[18,20,21]$} \\
\hline
\end{tabular}

Weber problem with opening costs of setting up facilities. The authors designed a new nonlinear mathematical model with rectilinear and Euclidean distances and proposed Variable Neighborhood Search (VNS) and Simulated Annealing (SA) to tackle the problem.

Table 1 summarizes previous studies on continuous LAP discussed in this section. Generally, three solution methods have been used to solve the problem. Most studies focused on continuous LAP without involving costs. However, continuous LAP with fixed cost is more practical and closer to the real application of LAP. Studies on this problem are still growing and the quality of new techniques can be tested on this problem. Due to this reason, this paper develops a hybrid heuristic algorithm based on CSBH and FDR to solve the problem.

\section{Mathematical Formulation}

This paper applies the mathematical model proposed by [26] to formulate the continuous capacitated multifacility Weber problem with fixed cost. The mathematical model for this problem is as follows.

$$
\operatorname{Minimize} \sum_{i=1}^{\bar{M}} \sum_{j=1}^{n} x_{i j} d\left(X_{i}, a_{j}\right)+\sum_{i=1}^{\bar{M}} f\left(X_{i}\right) z_{i}
$$

Subject to

$$
\begin{array}{ll}
\sum_{i=1}^{\bar{M}} x_{i j}=w_{j}, & j=1, \ldots, n \\
\sum_{j=1}^{n} x_{i j} \leq b z_{i}, & i=1, \ldots, \bar{M} \\
x_{i j} \geq 0, & i=1, \ldots, \bar{M} ; j=1 \ldots, n \\
z_{i}=\{0,1\}, & i=1, \ldots, \bar{M}
\end{array}
$$

where,

$\bar{M}$ : an upper bound on the number of facilities to be located.

$n$ : the number of fixed demand points (or customer points).

$z_{i}=1$ if the $i$ th facility is opened, 0 otherwise, $i=1, \ldots, \bar{M}$.

$x_{i j}$ : quantity assigned from facility $i$ to customer $j, i=1, \ldots, c ; j=1, \ldots, n$.

$d(X, Y)$ : the Euclidean distance between locations $X$ and $Y, X, Y \in \Re^{2}$.

$X_{i}=\left(X_{i}^{1}, X_{i}^{2}\right)$ : coordinates of facility $i$ where $X_{i} \in \Re^{2}$.

$a_{j}=\left(a_{j}^{1}, a_{j}^{2}\right)$ : location of customer $j$ where $a_{j} \in \Re^{2},(j=1, \ldots, n)$.

$f(X)$ : the fixed cost to open a facility at $X \in \Re^{2}$.

$w_{j}$ : demand or weight of customer $j(j=1, \ldots, n)$.

$b$ : a fixed capacity of a facility, where $b \in \check{c}, \check{c}$ is a set of capacity facility.

The objective function (3.1) is to minimize the sum of transportation and the fixed costs. Constraint (3.2) is to ensure that the total demand of each customer is satisfied. Constraint (3.3) guarantees the capacity limits 
of the facilities are not exceeded. Constraint (3.4) is the non-negativity constraints and constraint (3.5) is the binary constraints. Note that $\sum_{i=1}^{\bar{M}} z_{i}$ represents the number of open facilities.

\section{THE HYBRID HEURISTIC ALGORITHM}

A combination of intelligent cell-based approach and FDR is proposed for solving this problem. A cell-based approach adopted from [15] is enhanced to find most or maybe all optimal locations of facilities from a smaller set of promising locations. It is called Cell Selection-based Heuristic (CSBH). The heuristic algorithm is enhanced to intelligently select profitable area that can increase the possibility of finding better locations.

The CSBH is hybridized with FDR to forbid the new selected locations to be close to the previous locations by selecting initial locations which are far apart from each other. The reasoning behind the FDR is to avoid from producing similar solutions to the previous ones i.e. if new potential locations are too close to the location(s) that were already selected in the previous iterations. Therefore, hybridization of both techniques is proposed to improve the selection of initial facility locations. The selection only occurred in the profitable areas and far apart from each facility location in order to minimize transportation cost. If profitable areas are never been considered to locate the facilities and the facilities are located close to each other, then it may increase the total transportation cost. In order to overcome this problem, an approach that can find profitable area intelligently is required. The proposed hybrid heuristic employed Cooper's ATL Heuristic, CSBH, and FDR; whereby its hybridization is explained in the following subsections.

\subsection{Cooper's ATL algorithm}

The proposed solution methodology is based on Cooper's ATL algorithm which is integrated into this problem. Cooper's ATL algorithm is an efficient multi-start iterative scheme that has short convergence time to find the best facility locations in continuous space based on the Weiszfeld equation (4.1). Kuhn [22] proved that the ATL algorithm with the Euclidean distance found its global minimum for each facility and its subset of allocated customers though it can only guarantee a local minimum. The ATL heuristic algorithm has been successfully implemented to find initial facility configurations or to deal with the capacitated multi-facility Weber problem and its variants, see $[19,27-29,33,42]$.

Based on Cooper's ATL algorithm, $M$ open facilities are chosen from the fixed points of customer locations at the beginning. Then, the $M$ open facilities are used to obtain the allocation for this capacitated problem. The output is the $M$ independent set of allocations which each subset consist of $n_{i}$ fixed points where $i=1,2, \ldots, M$ and $\sum n_{i} \geq n$. In the allocation stage, some customers may have their demand split between different facilities as the customers may have more than one demand, thus the symbol " $\geq$ " is used instead of "=" symbol. Then, an iterative procedure based on the Weiszfeld equations as given in equation (4.1) is applied to find the new location of each $M$ facility $(i=1,2, \ldots, M)$.

$$
\begin{aligned}
X_{i}^{1^{(k)}} & =\frac{\sum_{j_{i}=1}^{n_{i}} \frac{w_{j_{i}} a_{j_{i}}^{1}}{d\left(X_{i}^{(k-1)}, a_{j_{i}}\right)}}{\sum_{j_{i}=1}^{n_{i}} \frac{w_{j_{i}}}{d\left(X_{i}^{(k-1)}, a_{j_{i}}\right)}} \text { and } \\
X_{i}^{2^{(k)}}= & \frac{\sum_{j_{i}=1}^{n_{i}} \frac{w_{j_{i}} a_{j_{i}}^{1}}{d\left(X_{i}^{(k-1)}, a_{j_{i}}\right)}}{\sum_{j_{i}=1}^{n_{i}} \frac{w_{j_{i}}}{d\left(X_{i}^{(k-1)}, a_{j_{i}}\right)}}
\end{aligned}
$$

where the superscript $k$ is the iteration number within the Weiszfeld iterative procedure; $\left(a_{j_{i}}^{1}, a_{j_{i}}^{2}\right)$ represents the location of the $j_{i}$ th fixed points where $j_{i}=1,2, \ldots, n_{i} ;\left(X_{i}^{1^{(k)}}, X_{i}^{2^{(k)}}\right)$ denotes the new location of the $i$ th facility at iteration $k(i=1,2, \ldots, M)$ and $w_{j_{i}}$ corresponds to all or a fraction of the $j$ th customer demand that is served by facility $i$. The demand of some customers might have been split as a result of the solution of the 
transportation problem (i.e. $w_{j_{i}} \leq w_{j}$ ). Therefore, some customers may be utilized more than once in equation (4.1) but each time only with a portion $w_{j_{i}}$ of their demand. Then, the process of alternating between the LAP and transportation problem is applied until no improvement in total cost can be found. The main steps of Cooper's ATL Heuristic are presented in Algorithm 1:

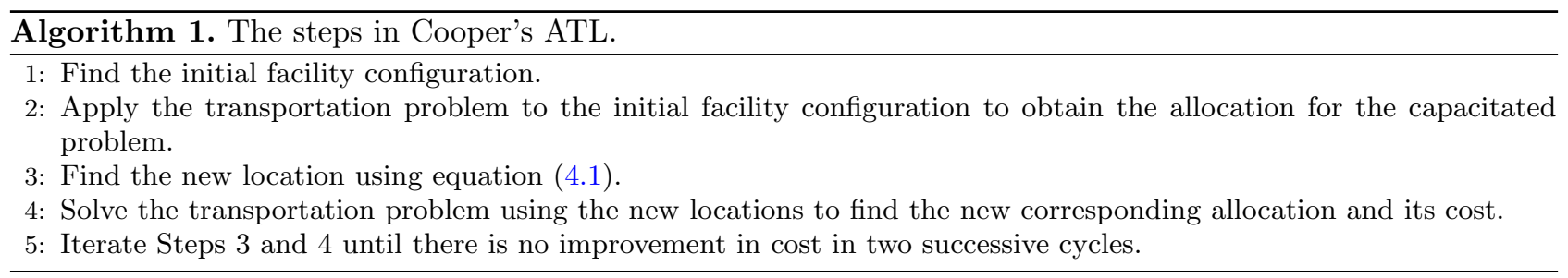

In Step 1, points of customer locations as the initial facility configuration are selected randomly. After that, transporation problem is applied to allocate customers to the nearest facility. New facilities are obtained using Weiszfeld equation (4.1) for the next step and the transportation problem is applied once again for the new selected facilities. This study also proposes the idea to restrict generating initial location that is close to the previously found locations. The method is reiterated several times using different random starting locations to diversify the search and increase the chance of reaching a near optimal solution.

\subsection{Cell Selection-based Heuristic (CSBH)}

The CSBH is a constructive heuristic technique that is modified from cell-based approach proposed by [14] which discretizes the continuous plane into a fixed number of equal size cells. This technique is used to generate facilities locations efficiently by selecting most or maybe all of the optimal sites of facilities locations from a smaller set of promising potential locations. In the newly proposed CSBH, facilities locations are generated in the profitable cells only and the unprofitable cells are discarded. This feature differs from the cell-based approach as it was not considered as a strategy in the past. In this study, CSBH is used to generate initial facility configuration for the CCLAP. Algorithm 2 shows the steps to construct CSBH that acts to divide the plane into cells in order to improve the selection of the facilities location.

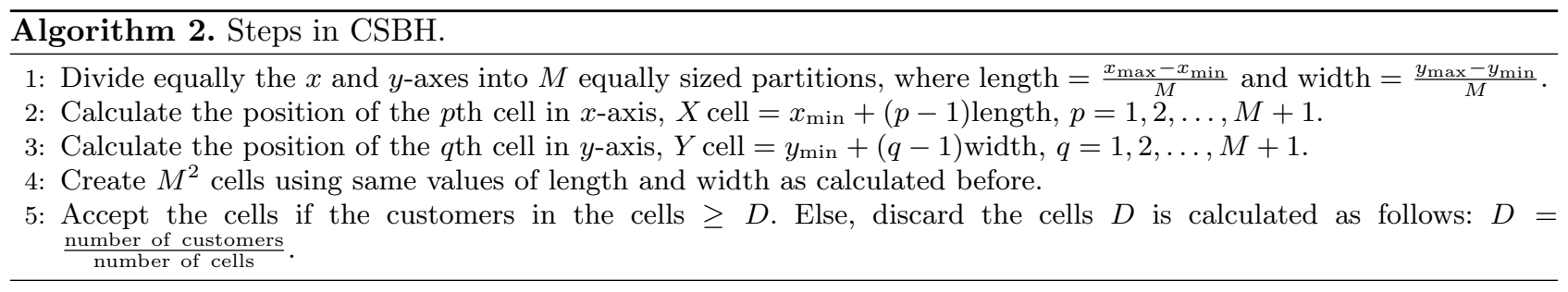

First, the plane is divided into $M^{2}$ equally sized partitions by using the formula in Step 1 to find the length and width of the cells. Then, the cells consist of less number of customers or unprofitable cells are discarded. The number of customers need to be in a cell is determined by the formula in Step 5 . The average number of customers in the cells is considered to identify how many cells to be discarded instead of the average demands of customers in the cells. This is because there are possibilities that higher number of demands is neglected in the unselected areas when the problem deals with multiple demands from each customer that lead to higher average demands. The demands from unselected areas will be served by the farthest facilities and it will result higher transportation costs. 


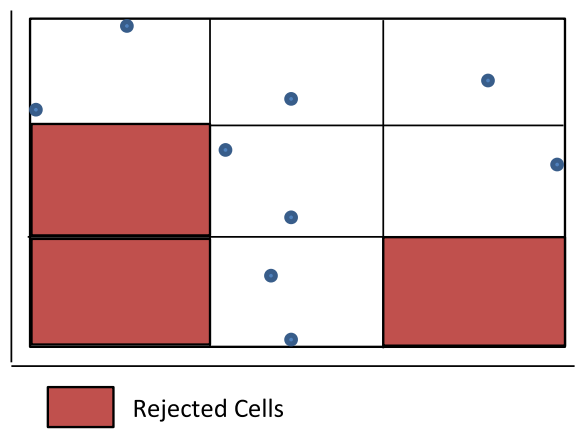

FIGURE 1. An illustrative of cell selection.

Figure 1 illustrates the cell selection process. First, let us consider to establish 3 facilities $(M=3)$ and the overall plane is divided into 9 cells $\left(M^{2}\right)$. Then, cells that consist of less than 1 customer are discarded. This is shown by using the formula in Step 5 in Algorithm 2 where 9 customers are divided by 9 cells, thus supposedly 1 cell needs to have 1 customer or more. The unshaded areas in Figure 1 show that cells with 1 customer or more will be considered to be selected to locate facilities.

\subsection{Furthest Distance Rule (FDR)}

The FDR is used to generate reasonably quicker initial facilities locations which are far apart from each other. This method takes into account the already found facilities locations while generating the new facilities locations. The first facility location is chosen randomly from the existing fixed locations, then the remaining facilities locations are generated using equation (4.2) as follows:

$$
\sum_{i \in E_{1}} d\left(a_{j *}, X_{i}\right)=\max _{(j \in J)} \sum_{i \in E_{1}} d\left(a_{j *}, X_{i}\right)
$$

Let $a_{j}=\left(a_{j}^{1}, a_{j}^{2}\right)$, be the location of customer $j$.

$X_{i}=\left(X_{i}^{1}, X_{i}^{2}\right)$, be the coordinates of the facility $i$.

$E_{1}=$ a set of initial facilities locations.

$D\left(X_{i}, a_{j}\right)=$ the Euclidean distance between facility $i$ and customer $j$.

$a_{j *}=$ the furthest point from the initial solutions which have been chosen previously.

Let $p$ be the number of facilities to be located, and $E_{1}$ be a set of initial facility locations. The main steps in FDR method are shown in Algorithm 3.

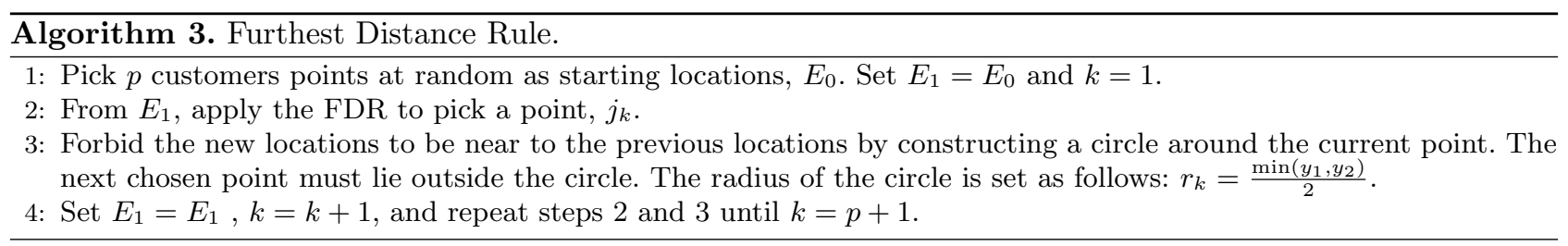

First facility is picked randomly and the next facility is selected using FDR where the farthest facility is chosen from two random points of the potential locations of the facility. At the same time, the radius of each facility is generated using the formula in Step 3 to forbid the new facilities to be near to the previous selected facilities. The points in the radius, $r_{k}$, are rejected to be the next facilities. 


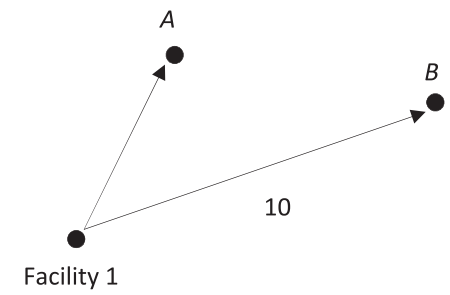

FIGURE 2. An illustrative of the first and second facility selections.

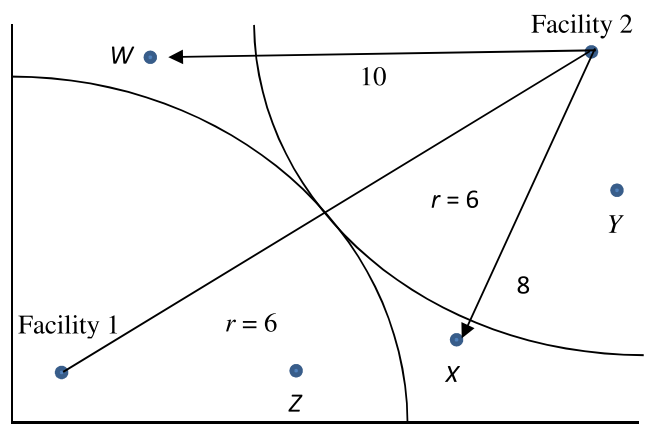

Figure 3. An illustrative of the third facility selection.

Figure 2 shows an illustration on how FDR is constructed. First, facility is selected randomly and then, two random points are generated as potential locations $A$ and $B$. The farthest point from the first facility is chosen as the second facility. As in Figure 2, potential location $B$ is farther when compared with potential location $A$. Thus, potential location $B$ is selected as the second facility location.

Figure 3 shows the process to select the third facility location. The radius of each facility point is constructed using the formula in Step 3 in Algorithm 3 to avoid the next facility to be near to the previous chosen facilities. The calculated radius $(r)$ shown in Figure 3 is 6 . The customer point which is the farthest from the second facility and located outside the radius of both previous facilities is selected as the third facility. As the selection of the second facility, two random points are also generated to select the third facility. The searches of the two random points are repeated if either one or both points are inside the radius. Only two points are randomly generated in every selection of the facility in order to save the searching time. Points $Y$ and $Z$ are rejected to be potential facility locations as shown in Figure 3 because both points are located inside the radius of facilities 1 and 2. Then, points $X$ and $W$ are generated for the next search and the points are suitable to be potential facility locations since they are outside of the calculated radius. Point $W$ has a longer distance from the second facility compared to point $X$. Thus, point $W$ is selected as the third facility location.

\subsection{The proposed hybrid heuristic algorithm}

The steps of the proposed hybridization algorithm are given in Algorithm 4. First, CSBH is constructed to generate $M^{2}$ equal cell and unprofitable cells are discarded in Step 1. The first facility location is selected randomly from customers' locations and the next facilities' locations are selected using the FDR in Step 2. Transportation problem is accordingly applied to find allocation of each customer to facilities and costs are calculated in Step 3. In Steps 4 and 5, the new facilities locations are generated using equation (4.1) and allocation of the customers and its cost is calculated. Lastly, Steps $2-5$ are repeated to obtain the lowest total cost as the final result until there is no further improvement between two successive iterations. 


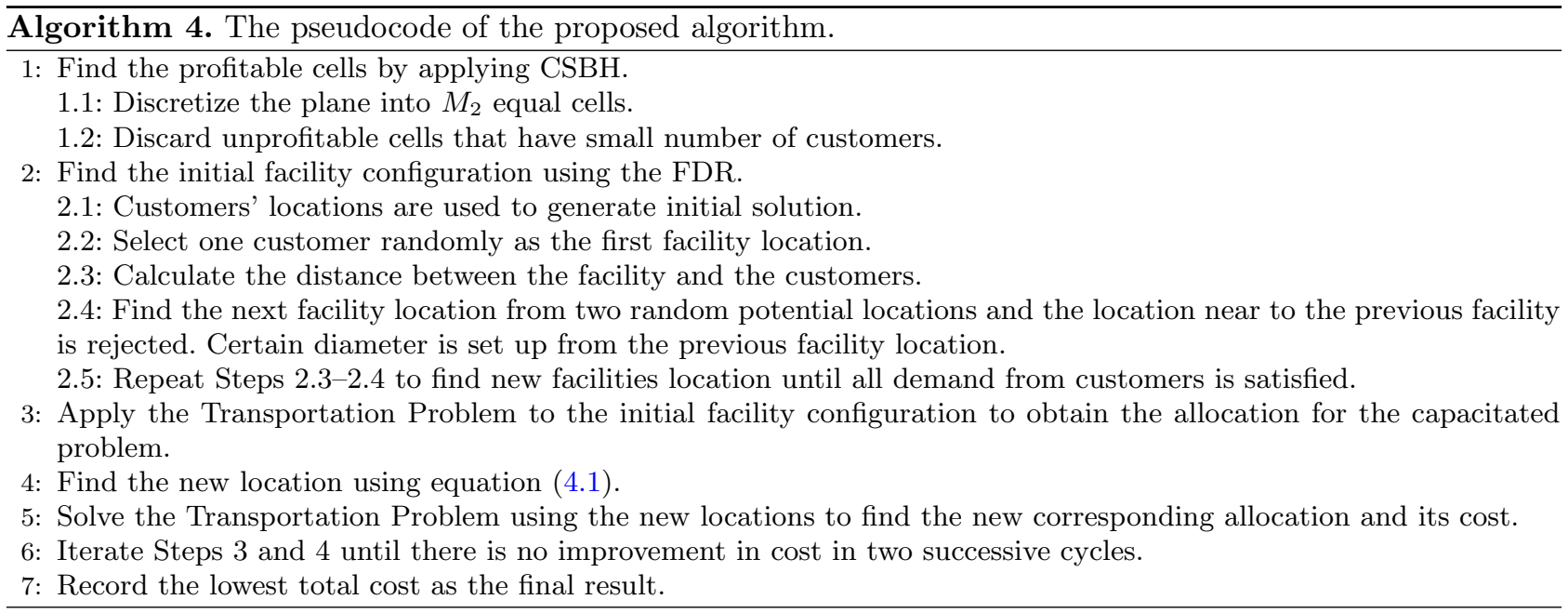

\section{EXPERIMENTS}

The performance of the proposed hybrid heuristic algorithm was evaluated using a benchmark dataset taken from [26] and the results were directly compared with other studies. The dataset was originally introduced by [8] for the uncapacitated problem. The datasets contained customers' locations represented as coordinates of $x$ and $y$-axis, demand of each customer and fixed cost of each customer's fixed point. Datasets of sizes 50, 287, 654, and 1060 fixed customers' locations are considered where three instances for size 50 and five instances for the other sizes with different values of capacities. This structure was used because 50 fixed points were considered as a small problem where the other three instances represented larger problems. However, the other datasets need to be tested on larger number of facilities as the datasets consisted of larger number of customers.

The lower bound (LB) of number of facility for every instance was set based on the capacity of the instances. LB is considered as the start number of facilities to find a facility configuration with the lowest total cost. In this case, small number of facilities may cause infeasibility during the solution search. This is because the total demand exceeded the total capacity of the facilities but was improved by starting the search using LB calculated as $\left[\sum_{j=1}^{n} w_{j} / b\right]$ as presented in [26]. The same LB formula presented in [26] was used in this study for consistency purpose. The total cost of lower bound facilities was also calculated and stated as initial total cost because smaller number of facilities generated lower total fixed costs. The search was terminated when the total cost increased for consecutive iterations.

The proposed hybrid heuristic algorithm was coded in $\mathrm{C}++$ programming and was compiled using Microsoft Visual Studio 2008 and ran on HP Intel@ Core ${ }^{\mathrm{TM}} \mathrm{i} 3-4030 \mathrm{U}$ CPU @ $1.90 \mathrm{GHz}$ computer with $4 \mathrm{~GB}$ RAM. It is worth noting that when dealing with larger problem sizes such as huge number of customers and multi-demand, the computation time increases exponentially. The experiment was repeated for 60 max_run for 50 customers, 80 max_run for 287 customers and 100 max_run for 654 and 1060 customer's data sets. These figures (number of runs of 60,80 , and 100) were found when the preliminary experiments were carried out. Then, the Weiszfeld algorithm was iterated for 1000 iterations and stopped after two non-improving iterations. The lowest total cost was then chosen as the best result and is highlighted in bold in Table 2.

\section{RESUlts COMPARISON AND DISCUSSION}

This section discusses and compares results obtained by CSBH, FDR, and the hybrid heuristic of CSBH and FRD with previous published results. The first comparison was made between the proposed hybrid heuristic algorithm and single CSBH and FDR algorithms in order to evaluate its performance. The comparison outcomes 
TABLE 2. Comparison of results between CSBH, FDR and hybrid heuristic algorithm.

\begin{tabular}{|c|c|c|c|c|c|c|c|c|}
\hline \multirow[b]{2}{*}{$\begin{array}{l}\text { Number of } \\
\text { customers }(n)\end{array}$} & \multirow[b]{2}{*}{$\begin{array}{l}\text { Capacity } \\
\text { (b) }\end{array}$} & \multirow[b]{2}{*}{$\begin{array}{l}\text { Lower } \\
\text { bound (LB) }\end{array}$} & \multicolumn{2}{|c|}{$\mathrm{CSBH}$} & \multicolumn{2}{|c|}{ FDR } & \multicolumn{2}{|c|}{ Hybrid CSBH-FDR } \\
\hline & & & $M^{*}$ & $\begin{array}{l}\text { Total cost } \\
\text { using } M^{*}\end{array}$ & $M^{*}$ & $\begin{array}{l}\text { Total cost } \\
\text { using } M^{*}\end{array}$ & $M^{*}$ & $\begin{array}{l}\text { Total cost } \\
\text { using } M^{*}\end{array}$ \\
\hline \multirow[t]{3}{*}{50} & 10 & 5 & 9 & 82.06 & 8 & 80.98 & 8 & $80.27 *$ \\
\hline & 5 & 10 & 14 & 81.45 & 13 & 79.11 & 13 & $79.11^{*}$ \\
\hline & 3 & 17 & 18 & 113.49 & 19 & 102.15 & 19 & $102.15^{*}$ \\
\hline \multirow[t]{5}{*}{287} & 1264 & 5 & 13 & 8513.04 & 12 & 8742.13 & 12 & $8338.45^{*}$ \\
\hline & 632 & 10 & 13 & 9000.21 & 12 & 9151.98 & 12 & $8918.17^{*}$ \\
\hline & 422 & 15 & 18 & 9638.10 & 16 & 9882.57 & 15 & $9618.52^{*}$ \\
\hline & 316 & 20 & 20 & 10142.58 & 20 & 10378.87 & 23 & $10206.02^{*}$ \\
\hline & 211 & 30 & 32 & 12401.98 & 30 & 12751.44 & 31 & $12578.53^{*}$ \\
\hline \multirow[t]{5}{*}{654} & 131 & 5 & 13 & 146804.71 & 12 & 156895.20 & 14 & $152028.70^{*}$ \\
\hline & 66 & 10 & 14 & 155970.28 & 15 & 158105.84 & 16 & $149171.35 *$ \\
\hline & 33 & 20 & 22 & 170834.47 & 23 & 181137.54 & 23 & $173785.61^{*}$ \\
\hline & 22 & 30 & 31 & 214202.63 & 31 & 218676.32 & 31 & $210546.00^{*}$ \\
\hline & 17 & 40 & 42 & 236169.45 & 41 & 240130.08 & 40 & $233217.93^{*}$ \\
\hline \multirow[t]{5}{*}{1060} & 212 & 5 & 11 & 1527288.32 & 11 & 1629629.24 & 11 & $1589850.93^{*}$ \\
\hline & 106 & 10 & 11 & 1585639.09 & 11 & 1596249.21 & 11 & $1533971.03^{*}$ \\
\hline & 53 & 20 & 22 & 1579471.29 & 21 & 1696776.59 & 22 & $1684427.68^{*}$ \\
\hline & 36 & 30 & 32 & 1867194.34 & 31 & $1795211.26 *$ & 32 & 1875931.34 \\
\hline & 27 & 40 & 41 & 2143998.12 & 40 & $2096987.78^{*}$ & 41 & 2136121.59 \\
\hline
\end{tabular}

are presented in Table 2. Most of the instances (11 out of 18) showed that the results of hybrid heuristic are superior to the ones obtained by single CSBH and FDR algorithms. The hybrid heuristic produced better results than single CSBH for 12 out of 18 instances (the better one is in italic). Some of the results using hybrid heuristic are less inferior to the CSBH results. This is due to the radius setting in the hybrid heuristic algorithm, which prevented the facilities not to be nearer to each other and this situation could happen when a high number of customers is concentrated in one area and not sparsely distributed. On the other hand, the result of hybrid heuristic is also better than the single FDR for 16 out of 18 instances (the better ones are indicated with asterisk). The last two instances for 1060 customers are the only solutions which show that single FDR is better than the hybrid heuristic. In this case, it might show that the hybrid heuristic did not perform well for large problem size in these instances. This is ascribed to the formula used in finding the profitable areas in the hybrid heuristic upon considering that all areas as profitable whenever the problem size increased. This could happen when defining the size of profitable areas for large instances are too loose and hence some profitable areas may be neglected. To overcome this, one can redefine the size of profitable areas when the problem size increases using a certain threshold based on a number of customers and a capacity of a facility.

We also conducted a comparative study between our results and the results presented in [26] since both studies applied similar structure and datasets. Table 3 shows the comparison of results between GRASP proposed by [26] and our proposed hybrid heuristic. The results showed that the hybrid heuristic displayed similar performance with the GRASP. The first two instances for size 50 were better than [26]. The strategic locations of the facilities where most of the customers' location was nearer to the facilities location, showed that the algorithm performed well and this minimize the total cost.

The problem size of 287 differed from other data sets as the demands were not set to unity. The results from all five instances of size 287 were not as good as the results of GRASP. This is because the proposed hybrid heuristic divides the distribution of customers into cells or small areas. In these data sets, the sparsity of customers was very close and concentrated in a few areas only. On the other hand, the total demand of a few cells was higher than the other cells. The demand that cannot be fulfilled by the assigned facilities in the cells 
TABLE 3. Comparison of results among GRASP and hybridization of CSBH and FDR.

\begin{tabular}{|c|c|c|c|c|c|c|c|c|c|c|c|}
\hline \multirow[b]{2}{*}{$\begin{array}{l}\text { Number of } \\
\text { customers } \\
(n)\end{array}$} & \multirow[b]{2}{*}{$\begin{array}{l}\text { Capacity } \\
(b)\end{array}$} & \multirow[b]{2}{*}{$\begin{array}{l}\text { Lower } \\
\text { bound } \\
\text { (LB) }\end{array}$} & \multicolumn{4}{|l|}{ GRASP } & \multicolumn{4}{|c|}{ Hybrid CSBH-FDR } & \multirow{2}{*}{$\begin{array}{l}\% \\
\text { Improve }\end{array}$} \\
\hline & & & $\begin{array}{l}\text { Total cost } \\
\text { using } \\
\text { LB }\end{array}$ & $M^{*}$ & $\begin{array}{l}\text { Total cost } \\
\text { using } \\
M^{*}\end{array}$ & $\begin{array}{l}\mathrm{CPU} \\
(\mathrm{s})\end{array}$ & $\begin{array}{l}\text { Total cost } \\
\text { using } \\
\text { LB }\end{array}$ & $M^{*}$ & $\begin{array}{l}\text { Total cost } \\
\text { using } \\
M^{*}\end{array}$ & $\begin{array}{l}\mathrm{CPU} \\
(\mathrm{s})\end{array}$ & \\
\hline \multirow[t]{3}{*}{50} & 10 & 5 & 95.05 & 8 & 81.09 & 13 & 102.94 & 8 & 80.27 & 0 & +1.01 \\
\hline & 5 & 10 & 91.68 & 13 & 81.52 & 32 & 94.54 & 13 & 79.11 & 1 & +2.96 \\
\hline & 3 & 17 & 97.33 & $17^{*}$ & 97.33 & 62 & 108.74 & 19 & 102.15 & 1 & -4.95 \\
\hline \multirow[t]{5}{*}{287} & 1264 & 5 & 11738.38 & 12 & 8180.17 & 294 & 11719.35 & 12 & 8338.45 & 2 & -1.93 \\
\hline & 632 & 10 & 10306.11 & 13 & 8516.38 & 290 & 9727.48 & $12^{*}$ & 8918.17 & 2 & -4.72 \\
\hline & 422 & 15 & 9307.46 & 18 & 8571.86 & 752 & 9682.38 & $15^{*}$ & 9618.52 & 3 & -12.21 \\
\hline & 316 & 20 & 9348.14 & 26 & 8907.67 & 2976 & 10592.06 & $23^{*}$ & 10206.02 & 2 & -14.58 \\
\hline & 211 & 30 & 10801.66 & 32 & 10607.88 & 3318 & 11638.14 & $31^{*}$ & 12578.53 & 4 & -18.58 \\
\hline \multirow[t]{5}{*}{654} & 131 & 5 & 346332.01 & 14 & 149929.33 & 1341 & 344362.16 & 14 & 152028.70 & 10 & -1.40 \\
\hline & 66 & 10 & 221651.96 & $15^{*}$ & 149183.10 & 1388 & 221752.71 & 16 & 149171.35 & 20 & +0.01 \\
\hline & 33 & 20 & 209650.64 & 23 & 182340.27 & 4526 & 197221.28 & 23 & 173785.61 & 32 & +4.69 \\
\hline & 22 & 30 & 224657.43 & 34 & 214481.92 & 16466 & 235050.24 & $31^{*}$ & 210546.00 & 36 & +1.84 \\
\hline & 17 & 40 & 239132.80 & 40 & 239132.80 & 10401 & 244500.03 & 40 & 233217.93 & 37 & +2.47 \\
\hline \multirow[t]{5}{*}{1060} & 212 & 5 & 2154044.58 & 11 & 1569610.77 & 2866 & 2123022.28 & 11 & 1589850.93 & 10 & -1.29 \\
\hline & 106 & 10 & 1673323.58 & 12 & 1606542.46 & 1798 & 1731338.54 & $11^{*}$ & 1533971.03 & 29 & +4.52 \\
\hline & 53 & 20 & 1672401.91 & $20^{*}$ & 1672401.91 & 3591 & 1752226.44 & 22 & 1684427.68 & 41 & -0.72 \\
\hline & 36 & 30 & 1964836.78 & $30^{*}$ & 1964836.78 & 10417 & 1947707.27 & 32 & 1875931.34 & 53 & +4.52 \\
\hline & 27 & 40 & 2184908.54 & $40^{*}$ & 2184908.54 & 22787 & 2235853.31 & 41 & 2136121.59 & 60 & 2.23 \\
\hline
\end{tabular}

were fulfilled by the other facilities in other cells. This led to higher transportation costs. However, the hybrid heuristic algorithm gave better results in terms of $M^{*}$ i.e. lower number of facilities compared to GRASP. Since the unprofitable areas were already discarded, the facilities were only located in the areas of higher number of customers and the number of facilities needed can be minimized to serve the customers.

The results for problem size of 654 showed that the proposed hybrid heuristic is superior to the GRASP for almost all instances. Even though the first instance for 654 customer points is inferior to the GRASP, the results are better for the next remaining instances as the number of facilities increased. On the other hand, most of the instances displayed better results of $M^{*}$ or equal performance when compared with the results produced by [26] except for the second instance.

The results for problem size 1060 showed that the first and third instances of this dataset consisted of 212 and 53 capacities, which were not as good as the results produced by GRASP where the GRASP is only better than the hybrid heuristic at $1.29 \%$ and $0.71 \%$ improvement, respectively. The other instances showed that the hybrid heuristic performed better than the GRASP up to $4.52 \%$ improvement. Even though this problem introduced fixed costs in the datasets, the transportation costs were minimized when many facilities were involved. The last three instances obtained higher number of facilities due to the decrement of the capacity limit.

However, the last instance showed that [26] recorded better results up to $4.95 \%$ because the facilities were located nearer to each other, in which the facilities could not serve the customers effectively. Moreover, this study obtained the same number of $M^{*}$ except for the last instance with higher number of facilities than the previous study as the limit of the capacity decreased. This is because certain cells consisted of higher number of customers thus, more facilities were needed to serve the customers in that areas due to lower capacity limit.

Overall results from the proposed hybrid heuristic showed that 9 out of 18 instances gave better results compared to the results from [26]. Hence, it can be concluded that the hybrid heuristic algorithm can solve the problem efficiently especially for large size problem. When the size of the problem is larger (involved more facilities), the results produce better solutions. These are proven in the results of 654 and 1060 customers. Moreover, this study exemplified better results when customer demand was set to unity. 


\section{Conclusion}

This paper presents a solution approach for solving the CCLAP with continuous fixed costs. A hybrid heuristic of the CSBH and the FDR was developed and enhanced to address the problem. The proposed hybrid heuristic could divide the distribution of customers into smaller set of promising locations and intelligently select profitable area to increase the possibility of finding better locations. The new locations of the facilities are forbidden to be close to the previously selected locations by using FDR. Various sizes of problems (50, 287, 654, and 1060 customers) with different values of capacities were used to assess the performance of the proposed hybrid heuristic. The experiments showed that the hybrid heuristic performed better than the single CSBH and FDR; displaying competitive results when compared with the previous published results. Hence, this study proves that this hybridization heuristic of CSBH and FDR could generate competitive results by producing deviation of less than $4.92 \%$ with average $3.92 \%$ compared to the results reported in [26]. This is applied to most of large problem sizes that had higher number of facilities. Besides, the proposed hybrid heuristic algorithm obtained better results for the problems where there is only one demand for each customer. However, for the situation of customers with more than one demand or multiple demands, the proposed hybrid heuristic algorithm failed to show much improvement. Thus, this study can be extended and improved by implementing metaheuristics techniques such as population search-based approaches (e.g. Genetic algorithm, ant colony optimization, and particle swam optimization) to obtain better solutions and to ensure that the solution search can be extensively done especially for problems with multiple demand. One can refer to [10] for more details on such techniques to solve continuous LAP.

Acknowledgements. The authors sincerely acknowledge the reviewers for the constructive feedback and valuable comments.

\section{REFERENCES}

[1] M. Akyüz, I.K. Altinel and T. Öncan, Location and allocation based branch and bound algorithms for the capacitated multifacility Weber problem. Ann. Oper. Res. 222 (2014) 45-71.

[2] M. Alizadeh, I. Mahdavi, S. Shiripour and H. Asadi, A nonlinear model for a capacitated location-allocation problem with Bernoulli demand using sub-sources. Int. J. Eng. Trans. B: App. 26 (2013) 1007-1016.

[3] M. Alizadeh, I. Mahdavi, N. Mahdavi-Amiri and S. Shiripour, A capacitated location-allocation problem with stochastic demands using sub-sources: an empirical study. Appl. Soft Comput. 34 (2015) 551-571.

[4] N. Aras, I.K. Altinel and M. Orbay, New heuristic methods for the capacitated multi-facility Weber problem. Nav. Res. Logist. 54 (2007) 21-32.

[5] N. Aras, S. Yumusak and I.K. Altinel, Solving the capacitated multi-facility Weber problem by simulated annealing, threshold accepting and genetic algorithms, edited by K.F. Doerner, M. Gendreau, P. Greistorfer, W. Gutjahr, R.F. Hartl and M. Reimann. In: Metaheuristics: Progress in Complex Systems Optimization. Springer, US (2007) 91-112.

[6] N. Aras, M. Orbay and I.K. Altinel, Efficient heuristics for the rectilinear distance capacitated multi-facility Weber problem. J. Oper. Res. Soc. 59 (2008) 64-79.

[7] J. Brimberg and S. Salhi, A continuous location-allocation problem with zone-dependent fixed cost. Ann. Oper. Res. 136 (2005) 99-115.

[8] J. Brimberg, P. Hansen, N. Mladenović and E.D. Taillard, Improvements and comparison of heuristics for solving the uncapacitated multisource Weber problem. Oper. Res. 48 (2000) 444-460.

[9] J. Brimberg, N. Mladenović and S. Salhi, The multi-source Weber problem with constant opening cost. J. Oper. Res. Soc. 55 (2004) 640-646.

[10] J. Brimberg, P. Hansen, N. Mladenović and S. Salhi, A survey of solution methods for the continuous location-allocation problem. Int. J. Oper. Res. 5 (2018) 1-12.

[11] L. Cooper, The transportation-location problem. Oper. Res. 20 (1972) 94-108.

[12] M. Daskin, Network and Discrete Location - Models, Algorithms and Applications. John Wiley \& Sons, Inc. (1995).

[13] A. Elalouf, D. Tsadikovich and S. Hovav, Optimization of blood sample collection with timing and quality constraints. Int. Trans. Oper. Res. 25 (2018) 191-214.

[14] M.D.H. Gamal and S. Salhi, Constructive heuristics for the uncapacitated continuous location-allocation problem. J. Oper. Res. Soc. 52 (2001) 821-829.

[15] M. Gamal and S. Salhi, A cellular heuristic for the multisource Weber problem. Comput. Oper. Res. 30 (2003) $1609-1624$.

[16] P. Hansen, N. Mladenović and E. Taillard, Heuristic solution of the multisource Weber problem as a $p$-median problem. Oper. Res. Lett. 22 (1998) 55-62. 
[17] S. J. Hosseininezhad, M.S. Jabalameli and S.G.J. Naini, A fuzzy algorithm for continuous capacitated location allocation model with risk consideration. Appl. Math. Model. 38 (2014) 983-1000.

[18] S.J. Hosseininezhad, S. Salhi and M.S. Jabalameli, A cross entropy-based heuristic for the capacitated multi-source Weber problem with facility fixed cost. Comput. Ind. Eng. 83 (2015) 151-158.

[19] C.A. Irawan, S. Salhi, M. Luis and N. Azizi, The continuous single source location problem with capacity and zone-dependent fixed cost: models and solution approaches. Eur. J. Oper. Res. 263 (2017) 94-107.

[20] C.A. Irawan, M. Luis, S. Salhi and A. Imran, The incorporation of fixed cost and multilevel capacities into the discrete and continuous single source capacitated facility location problem. Ann. Oper. Res. 275 (2019) 367-392.

[21] C.A. Irawan, S. Salhi and K. Soemadi, The continuous single-source capacitated multi-facility Weber problem with setup costs: formulation and solution methods. J. Glob. Optim. 78 (2020) 271-294.

[22] H.W. Kuhn, A note on Fermat's problem. Math. Program. 4 (1973) 98-107.

[23] C.L. Lara, F. Trespalacios and I.E. Grossmann, Global optimization algorithm for capacitated multi-facility continuous locationallocation problems. J. Glob. Optim. 71 (2018) 871-889.

[24] M. Luis, S. Salhi and G. Nagy, Region-rejection based heuristics for the capacitated multi-source Weber problem. Comput. Oper. Res. 36 (2009) 2007-2017.

[25] M. Luis, S. Salhi and G. Nagy, A guided reactive GRASP for the capacitated multi-source Weber problem. Comput. Oper. Res. 38 (2011) 1014-1024.

[26] M. Luis, S. Salhi and G. Nagy, A constructive method and a guided hybrid grasp for the capacitated multi-source Weber problem in the presence of fixed cost. J. Algorithms Comput. Tech. 9 (2015) 215-232.

[27] M. Luis, C. Irawan and A. Imran, A two-stage method for the capacitated multi-facility location-allocation problem. Int. J. Oper. Res. 35 (2019) 366-377.

[28] H. Manzour, A. Torabi and M.S. Pishvaee, New heuristic methods for the single-source capacitated multi facility Weber problem. Int. J. Adv. Manuf. Technol. 69 (2013) 1569-1579.

[29] S.M.H. Manzour-al-Ajdad, S.A. Torabi and K. Eshghi, Single-source capacitated multi-facility Weber problem - an iterative two phase heuristic algorithm. Comput. Oper. Res. 39 (2012) 1465-1476.

[30] N. Mohammadi, M.R. Malek and A.A. Alesheikh, A new GA based solution for capacitated multi source Weber problem. Int. J. Comput. Intell. Syst. 3 (2010) 514-521.

[31] S.M. Mousavi and S.T.A. Niaki, Capacitated location allocation problem with stochastic location and fuzzy demand: a hybrid algorithm. Appl. Math. Model. 37 (2013) 5109-5119.

[32] M. Mousazadeh, S.A. Torabi, M.S. Pishvaee and F. Abolhassani, Health service network design: a robust possibilistic approach. Int. Trans. Oper. Res. 25 (2018) 337-373.

[33] T. Öncan, Heuristics for the single source capacitated multi-facility Weber problem. Comput. Ind. Eng. 64 (2013) 959-971.

[34] D. Ozgen and B. Gulsun, Combining possibilistic linear programming and fuzzy AHP for solving the multi-objective capacitated multi-facility location problem. Inf. Sci. 268 (2014) 185-201.

[35] H.D. Sherali and C.H. Tuncbilek, A squared-euclidean distance location-allocation problem. Nav. Res. Logist. 39 (1992) 447469.

[36] H.D. Sherali, S. Ramachandran and S. Kim, A localization and reformulation discrete programming approach for the rectilinear distance location-allocation problem. Discrete Appl. Math. 49 (1994) 357-378.

[37] H.D. Sherali, I. Al-Loughani and S. Subramanian, Global optimization procedures for the capacitated euclidean and $l_{p}$ distance multifacility location-allocation problems. Oper. Res. 50 (2002) 433-448.

[38] C. Singhtaun and P. Charnsethikul, Efficient heuristics for single-source capacitated multi-facility Weber problems. In: Proceedings of the 38th International Conference on Computers and Industrial Engineering (2008).

[39] E.B. Tirkolaee, J. Mahmoodkhani, M.R. Bourani and R. Tavakkoli-Moghaddam, A self-learning particle swarm optimization for robust multi-echelon capacitated location-allocation-inventory problem. Int. J. Adv. Manuf. Technol. 18 (2019) 677-694.

[40] E.B. Tirkolaee, I. Mahdavi, M.M.S. Esfahani and G.-W. Weber, A robust green location-allocation-inventory problem to design an urban waste management system under uncertainty. Waste Manage. 102 (2020) 340-350.

[41] E.B. Tirkolaee, P. Abbasian and G.-W. Weber, Sustainable fuzzy multi-trip location-routing problem for medical waste management during the COVID-19 outbreak. Sci. Total Environ. 756 (2021) 143607.

[42] Z. Zainuddin and S. Salhi, A perturbation-based heuristic for the capacitated multisource Weber problem. Eur. J. Oper. Res. 179 (2007) 1194-1207.

[43] J. Zhou and B. Liu, New stochastic models for capacitated location-allocation problem. Comput. Ind. Eng. 45 (2003) 111-125. 